\title{
Social Patterning and Stability of Intention to Accept a COVID-19 Vaccine in Scotland: Will Those Most at Risk Accept a Vaccine?
}

\author{
Lynn Williams*(D), Paul Flowers (D), Julie McLeod ${ }^{D}$, David Young, Lesley Rollins and The CATALYST Project Team \\ School of Psychological Sciences \& Health, University of Strathclyde, Glasgow G1 1QE, UK; \\ paul.flowers@strath.ac.uk (P.F.); julie.mcleod.2020@uni.strath.ac.uk (J.M.); david.young@strath.ac.uk (D.Y.); \\ lesleyanne.rollins@strath.ac.uk (L.R.); behaviour-covid19@strath.ac.uk (C.P.T.) \\ * Correspondence: lynn.williams@strath.ac.uk
}

check for updates

Citation: Williams, L.; Flowers, P.; McLeod, J.; Young, D.; Rollins, L.;

The CATALYST Project Team. Social Patterning and Stability of Intention to Accept a COVID-19 Vaccine in Scotland: Will Those Most at Risk Accept a Vaccine. Vaccines 2021, 9, 17. https://doi.org/10.3390/ vaccines 9010017

Received: 19 November 2020 Accepted: 30 December 2020 Published: 4 January 2021

Publisher's Note: MDPI stays neutral with regard to jurisdictional clai$\mathrm{ms}$ in published maps and institutional affiliations.

Copyright: (C) 2021 by the authors. Licensee MDPI, Basel, Switzerland. This article is an open access article distributed under the terms and conditions of the Creative Commons Attribution (CC BY) license (https:// creativecommons.org/licenses/by/ $4.0 /)$.

\begin{abstract}
Vaccination is central to controlling COVID-19. Its success relies on having safe and effective vaccines and also on high levels of uptake by the public over time. Addressing questions of population-level acceptability, stability of acceptance, and sub-population variation in acceptability are imperative. Using a prospective design, a repeated measures two-wave online survey was conducted to assess key sociodemographic variables and intention to accept a COVID-19 vaccine. The first survey (Time 1) was completed by 3436 people during the period of national lockdown in Scotland and the second survey $(n=2016)$ was completed two months later (Time 2$)$ when restrictions had been eased. In the first survey, $74 \%$ reported being willing to receive a COVID-19 vaccine. Logistic regression analyses showed that there were clear sociodemographic differences in intention to accept a vaccine for COVID-19 with intention being higher in participants of white ethnicity as compared with Black, Asian, and minority ethnic (BAME) groups, and in those with higher income levels and higher education levels. Intention was also higher in those who had "shielding" status due to underlying medical conditions. Our results suggest that future interventions, such as mass media and social marketing, need to be targeted at a range of sub-populations and diverse communities.
\end{abstract}

Keywords: COVID-19; vaccination; vaccine hesitancy; interventions; social patterning; inequalities

\section{Introduction}

Vaccination will be vitally important in controlling future waves of the COVID-19 pandemic. Despite uncertainty regarding the specifics of many of the potential vaccines (e.g., efficacy and required doses), it is clear that high levels of overall public acceptance will be required. In recent years, vaccination rates have fallen and public confidence in vaccines has been inconsistent [1-4]. The term "vaccine hesitancy" refers to the "delay in acceptance or refusal of vaccines despite availability of vaccine services" $[5,6]$. The reasons for vaccine hesitancy are multi-levelled and complex, involving psychological, social, and contextual factors [7-9]. Vaccine hesitancy was evident during the H1NI pandemic, which saw variable vaccine uptake, with non-uptake related to concerns about vaccine safety and perceptions of threat and risk [10-14]. Preliminary evidence from the current pandemic suggests that a sizeable proportion of the public is currently either undecided or unwilling to receive a future vaccine for COVID-19 [15-20]. The importance of high levels of uptake was demonstrated in a recent study which suggested that in order to "extinguish an ongoing epidemic", the efficacy of a vaccine as the sole intervention needs to be at least $80 \%$ when uptake is at $75 \%$. If uptake is lower than this, then, an even more efficacious vaccine would be needed [21].

A recent global survey from Ipsos MORI [18], conducted in July and August 2020, involving 20,000 adults from 27 countries, found that $74 \%$ of people would get a vaccine if it was available. However, only $37 \%$ strongly agreed that they would want to get it, while $37 \%$ 
somewhat agreed. Large variations in acceptance levels were also shown across countries, ranging from $97 \%$ in China, to $54 \%$ in Russia. The primary reason that people gave for not wanting to accept the vaccine was worry about the side effects, followed by concern about the effectiveness of a vaccine, and not feeling at risk of contracting COVID-19 [18].

Emerging research has also suggested that particular sub-populations have lower acceptance intentions. In France, during late March 2020, 26\% of the sample reported that they would not accept a vaccine. COVID-19 vaccination hesitancy was more prevalent in those with low income, young women, and among older adults aged 75+, and it was associated with political views [19]. In Australia, more positive views were reported in April shortly after the introduction of lockdown, with $4.9 \%$ stating they would not get the vaccine, and 9.4\% stating indifference. Here, COVID-19 vaccination hesitancy was associated with lower education levels and health literacy, and the belief that the threat of COVID-19 had been exaggerated [20]. A study conducted two months later in Australia, when restrictions had been eased, found that those who were unwilling or unsure about accepting a COVID-19 vaccine had increased by 10\% [16], suggesting that COVID-19 vaccination levels may fluctuate depending on the context of infection rates and restrictions. A further study from the USA reported an acceptance level of $67 \%$ and found that acceptance levels were lower among unemployed participants and Black American participants [17].

Together, these initial studies suggest that COVID-19 vaccine acceptance differs both across countries and in different sub-populations within countries. The research to date has been based on cross-sectional designs and has not yet explored how the intention to accept a COVID-19 vaccine may change in line with rates of infection or lockdown restrictions within the same sample. We explored this question of intention to vaccinate against COVID-19 in a prospective survey in Scotland at two time points (during national lockdown and during the easing of restrictions). The present study has the following three key research questions (RQs):

RQ1 What proportion of people would accept a vaccine for COVID-19?

RQ2 Is COVID-19 vaccine acceptance stable over time in the context of different infection levels and restrictions?

RQ3 What sociodemographic factors are associated with intention to accept a future vaccine for COVID-19?

\section{Materials and Methods}

The present study consisted of an online survey at two time points. The first survey Time 1) was conducted during lockdown restrictions (from 20 May to 12 June 2020, weeks 9-12 of national lockdown). The second survey (Time 2) was conducted two months later (during August 2020) when lockdown restrictions in Scotland had been eased and there was little community transmission. Ethical approval was received from the University of Strathclyde Ethics Committee (ref. 61/05/05/2020/A Williams) prior to commencement of the study. The data reported here were part of the larger CATALYST project which examined the changes that people had experienced during lockdown.

\subsection{Participants and Procedure}

For Time 1, participants were recruited with convenience sampling through advertisements on social media, including Facebook and Twitter, which directed participants to Qualtrics where they could access the online questionnaire. Included in the survey for Time 1 was an information sheet and consent form which participants read and signed before completing the survey. For Time 2, participants were re-contacted via email and provided with a Qualtrics link to the online questionnaire, inviting them to take part in Time 2. 


\subsection{Questionnaire}

\subsubsection{Demographics and Health}

Participants provided self-reported sociodemographic and health data on the first survey for the following variables: age (18-49, 50+); gender (female, male); ethnicity (white, BAME); annual household income (<£16,000, £16,000-£29,999, £30,000-£59,999, £60,000+); level of education (no qualifications/left school at 16, high school/college, university); and risk group/shielding status (i.e., those who had been classified as high risk based on underlying health conditions or age) (yes/no).

\subsubsection{COVID-19 Vaccination Intention}

Intention to be vaccinated for COVID-19 was measured by self-reporting on the first survey and again on the second survey. Participants were asked "If a vaccine for Coronavirus (Covid-19) becomes available, would you want to receive it?" and provided response options "I definitely would not want to receive it", "I probably would not want to receive it", "unsure", "I probably would want to receive it" and "I definitely would want to receive it".

\subsection{Statistical Analysis}

The COVID-19 vaccination intention response options of "I definitely would not want to receive it", "I probably would not want to receive it", and "unsure" were coded as "vaccine hesitant" and the options "I probably would want to receive it" and "I definitely would want to receive it" were coded as "vaccine willing" to create a dichotomous "willing" versus "hesitant" variable. Frequency responses to the vaccination intention questions were calculated (RQ1). In addition, a McNemar's test was used to examine any changes in vaccine willingness across the two time points from lockdown (Time 1) to the easing of restrictions (Time 2) for those participants who completed the survey at both time points (RQ2). Univariate and multivariate logistic regression analyses were carried out to examine the sociodemographic factors associated with COVID-19 vaccination intention at Time 1 (we selected Time 1 intention as the dependent variable in order to maximise sample size) (RQ3). Due to issues relating to the representativeness of our sample and, specifically, the under-representation of males and those with lower levels of educational attainment (as shown in Table 1), we carried out post-stratification weighting of these variables based on the Scottish census data to correct for these imbalances. All of the analyses reported were based on this weighed data in order to enable greater extrapolation of the results to the Scottish population. All analyses were conducted using IBM SPSS Statistics (version 25) at $5 \%$ significant levels.

Table 1. Sociodemographic and health variables for the sample at survey one (Time 1) and survey two (Time 2).

\begin{tabular}{|c|c|c|c|c|}
\hline & Time 1 & & Time 2 & \\
\hline Variables & $\mathrm{n}$ & $\%$ & $\mathrm{n}$ & $\%$ \\
\hline \multicolumn{5}{|l|}{ Age } \\
\hline $18-49$ & 1847 & 53.8 & 974 & 48.3 \\
\hline $50+$ & 1578 & 45.9 & 1034 & 51.5 \\
\hline \multicolumn{5}{|l|}{ Gender } \\
\hline Female & 2719 & 79.1 & 1632 & 82.1 \\
\hline Male & 666 & 19.4 & 355 & 17.9 \\
\hline \multicolumn{5}{|l|}{ Ethnicity } \\
\hline White & 3308 & 96.3 & 1949 & 96.7 \\
\hline BAME & 101 & 2.9 & 52 & 2.6 \\
\hline \multicolumn{5}{|l|}{ Household income } \\
\hline$<£ 16,000$ & 334 & 9.7 & 196 & 9.7 \\
\hline$£ 16,000-£ 29,999$ & 611 & 17.8 & 348 & 17.3 \\
\hline$£ 30,000-£ 59,000$ & 1203 & 35.0 & 717 & 35.6 \\
\hline$£ 60,000+$ & 902 & 26.3 & 519 & 25.7 \\
\hline
\end{tabular}


Table 1. Cont.

\begin{tabular}{ccccc}
\hline & Time 1 & & Time 2 & \% \\
\hline Variables & $\mathbf{n}$ & $\mathbf{\%}$ & $\mathbf{n}$ & \\
\hline Education level & & & 77 & 3.8 \\
No quals/left school 16 & 168 & 4.9 & 439 & 7.8 \\
High school/college & 780 & 22.7 & 1467 & 72.8 \\
$\quad$ University & 2435 & 70.9 & 316 & 15.7 \\
High risk/shielding & & & 1677 & 83.2 \\
Yes & 508 & 14.8 & & \\
No & 2855 & 83.1 & & \\
\hline
\end{tabular}

Note, $\%$ calculations include missing data.

\section{Results}

Overall, 3436 participants (79\% female) residing in Scotland, aged over 18, were recruited for the current study. The sample was comprised of participants aged between 18 and $92(\mathrm{M}=46.21$ and $\mathrm{SD}=15.26)$. Of the original sample, 2016 participants took part in Time 2 , representing a 59\% follow-up rate. We compared the sociodemographic characteristics of those who participated in both waves of the survey with those who dropped out. There were no significant differences between the completers are non-completers based on ethnicity, income, or high-risk status but the groups did differ significantly on education level, gender, and age with higher dropout among those with lower education levels, males, and among the younger age group. Participant sociodemographic characteristics from Time 1 to Time 2 from our original sample (not weighted) are shown in Table 1.

\subsection{RQ1: What Proportion of People Would Accept a Vaccine for COVID-19?}

A frequency analysis of the proportion of participants that reported willingness and hesitancy to accept a COVID-19 vaccine across the two time points are shown in Table 2. At the time of the first survey, 74\% of participants were willing to receive a COVID-19 vaccine, and at the time of the second survey this figure was slightly higher at 78\% (note that the numbers reported in Table 2 for the "vaccine hesitant" and "vaccine willing" rows are based on the total sample, whereas the other numbers in the table are based upon only those participants who completed the questionnaire at both time points).

Table 2. Intention to accept a COVID-19 vaccine at Time 1 and Time 2.

\begin{tabular}{ccccc}
\hline COVID-19 Vaccine Intention & Time 1 (National Lockdown) & \multicolumn{2}{c}{ Time 2 (Easing of Restrictions) } \\
\hline I definitely would not want to receive it & $\mathbf{N}$ & \% & N & \% \\
I probably would not want to receive it & 54 & $3 \%$ & 65 & $4 \%$ \\
Unsure & 39 & $4 \%$ & 262 & $5 \%$ \\
I probably would want to receive it & 498 & $17 \%$ & 506 & $27 \%$ \\
I definitely would want to receive it & 904 & $49 \%$ & 919 & $50 \%$ \\
Vaccine hesitant & 850 & $26 \%$ & 416 & $22.5 \%$ \\
Vaccine willing & 2406 & $74 \%$ & 1433 & $77.5 \%$ \\
\hline
\end{tabular}

3.2. RQ2-Is COVID-19 Vaccine Acceptance Stable over Time in the Context of Different Infection Levels and Restrictions?

Table 2 presents the results relating to stability of COVID-19 vaccination intention for those participants who completed the questionnaire at both time points. The McNemar's test on these participants showed that there had been a significant shift in vaccine intention over time $(p=0.004)$. Among the 54 participants who reported that they definitely would not want to be vaccinated on Time 1, $13(24.1 \%)$ reported that they would now accept the vaccine on Time 2 . Among the 79 who reported that they probably would not want the 
vaccine on Time 1, 12 of them (15.2\%) reported they would now accept the vaccine on Time 2. Among the 301 who were unsure, $113(37.5 \%)$ reported on Time 2 that they would now accept the vaccine. Among the 904 people who initially would definitely want to be vaccinated, $20(2.2 \%)$ reported they would now not take it, and $10(1.1 \%)$ reported that they were now unsure. In addition, of the 498 who initially probably would receive it, 13 would now not take it $(2.6 \%)$, and 72 are now unsure $(14.5 \%)$.

\subsection{RQ3: What Sociodemographic and Health Factors Are Associated with Intention to Accept a Future Vaccine for COVID-19?}

As shown in Table 3, univariate logistic regression analyses showed that there was a significant effect of age, ethnicity, education level, household income, and high-risk/shielding status on intention to accept a COVID-19 vaccine. There was no effect of gender on intention to accept a COVID-19 vaccine. In relation to age, younger participants reported higher levels of intention than those in the older age group. We also found that participants of white ethnicity had higher levels of intention than those from the BAME groups. In relation to education, those with higher levels of education had higher levels of intention to receive the vaccine. In addition, those with higher levels of annual household income had higher intention levels than those with lower income levels. Finally, those participants at high risk or in the shielding category had higher levels of intention than those not in this group.

Table 3. Univariate analysis of sociodemographic factors and intention to accept a COVID-19 vaccine.

\begin{tabular}{ccccc}
\hline Variable & $p$-Value & Comparison & Coefficient & $p$-Value \\
\hline Age & $<0.001$ & $50+$ vs. 18-49 & 0.70 & - \\
Gender & 0.190 & Male vs. Female & 1.11 & - \\
Ethnicity & 0.018 & White vs. BAME & 1.98 & $<2$ \\
Education & $<0.001$ & High school/College vs. No & 2.78 & $<0.001$ \\
& & qualifications/left at 16 & 1.11 & $<.001$ \\
Household income & $<0.001$ & University vs. No qualifications /left at 16 & 1.39 & 0.441 \\
& & $£ 16,000-£ 29,999$ vs. $<£ 16,000$ & 1.97 & $<0.001$ \\
High risk/shielding & 0.012 & $£ 30,000-£ 59,999$ vs. $<£ 16,000$ & 1.31 & - \\
\hline
\end{tabular}

For the multivariate logistic regression, we entered those variables that were significant in the univariate analysis (i.e., age, ethnicity, education, household income, and high risk/shielding). Ethnicity, education, household income, and high risk remained significantly associated with intention to receive a COVID-19 vaccine in the multivariate analysis, but age was no longer significant. When considering the coefficients (see Table 4), those participants of white ethnicity were almost three times as likely to accept a COVID-19 vaccine as compared with those from BAME groups. Similarly, those from the highest education group were two and a half times more likely than those from the lowest education group to accept it, and those in the highest income group were 1.82 times more likely to accept the vaccine as compared with those in the lowest income group. In addition, those in a high-risk/shielding group were almost twice as likely to accept a COVID-19 vaccine as compared with those not in a high-risk/shielding group.

Table 4. Multivariate analysis of sociodemographic factors and intention to accept a COVID-19 vaccine.

\begin{tabular}{|c|c|c|c|c|c|}
\hline Variable & $p$-Value & Comparison & Coefficient & $95 \% \mathrm{CI}$ & $p$-Value \\
\hline Ethnicity & $<0.001$ & White vs. BAME & 2.91 & $1.75-4.81$ & - \\
\hline \multirow[t]{2}{*}{ Education } & $<0.001$ & $\begin{array}{l}\text { High school/College vs. no } \\
\text { qualifications/left at } 16\end{array}$ & 1.90 & $1.56-2.32$ & $<0.001$ \\
\hline & & $\begin{array}{l}\text { University vs. no } \\
\text { qualifications/left at } 16\end{array}$ & 2.50 & $1.95-3.21$ & $<0.001$ \\
\hline \multirow[t]{3}{*}{ Household income } & $<0.001$ & $£ 16,000-£ 29,999$ vs. $<£ 16,000$ & 1.05 & $0.80-1.38$ & 0.743 \\
\hline & & $£ 30,000-£ 59,999$ vs. $<£ 16,000$ & 1.27 & $0.98-1.65$ & 0.077 \\
\hline & & $£ 60,000+$ vs. $<£ 16,000$ & 1.82 & $1.35-2.45$ & $<0.001$ \\
\hline High risk/shielding & $<0.001$ & Yes vs. no & 1.95 & $1.53-2.49$ & - \\
\hline
\end{tabular}




\section{Discussion}

The present study is the first to examine intention to accept a COVID-19 vaccine in Scotland. The study also extends existing research that has examined acceptance levels in other countries by adopting a prospective design, allowing us to examine stability in vaccine intentions over time within the same sample. We found that $74 \%$ of the sample on Time 1 intended to receive a COVID-19 vaccine (during the period of national lockdown). Interestingly, two-months later, intention levels had shifted significantly. The greatest shift in intention levels was apparent in the group who were "unsure" at the time of the first survey, with $38 \%$ of those participants now saying they would accept a vaccine. The second survey was carried out when restrictions had been eased and there was little transmission of COVID-19, therefore, it is notable that intention to receive a COVID-19 vaccine was still high at this time. Stability in intention to receive a COVID-19 vaccination is particularly important given that two doses of the vaccine are likely to be needed over time.

Our findings suggested that although the majority of respondents in Scotland indicated that they would want to receive a COVID-19 vaccine, there was a sizeable minority of the public who were hesitant about receiving a vaccine, mirroring the overall picture that has been emerging from other countries. Indeed, the intention levels reported here are similar to those emerging globally from the recent Ipsos survey in which $74 \%$ of people overall said they would get a COVID-19 vaccine [18].

We also found that intention to accept a COVID-19 vaccination varied by sub-population. There was a significant effect of ethnicity, education level, household income, and highrisk/shielding status on intention to accept a COVID-19 vaccine. Of particular note, those of white ethnicity were nearly three times as likely to accept a COVID-19 vaccine as compared with those from BAME groups, those from the highest education group were two and a half times more likely than those from the lowest education group to accept it, and those in the highest income group were almost twice as likely to accept the vaccine as compared with those in the lowest income group.

Overall, those most at risk of the negative sequalae of COVID-19 had less intentions to get vaccinated than those at lower risk [22]. For example, intention was higher in participants of white ethnicity as comparison with the BAME groups. However, it should be noted that this binary approach to looking at differences in ethnicity is problematic, as it may mask important differences between diverse racialised and minoritised communities with distinct cultures and social norms. We also found that intentions for vaccination were higher in those with higher levels of income, again raising questions about why those most likely to suffer the negative consequences of COVID-19 would be the least likely to intend to vaccinate. These findings fit with the emerging literature on COVID-19 vaccine acceptance which has suggested that those from the BAME group may be less likely to accept a vaccine [17] and that COVID-19 vaccine hesitancy was more prevalent in those with lower income [19]. If these inequalities in intention translate into uptake, then, these findings are very important as they suggest the likelihood that vaccination programmes, unless implemented with targeted and tailored interventions to enhance vaccination rates, may actually amplify existing inequalities. More positively, intention to accept a COVID-19 vaccine was higher among those who were shielding (i.e., those who had been classified as high risk based on underlying health conditions or age).

Together, these initial studies highlight the need for government and public health bodies to think carefully about how to approach their publics with further demands for behavioural change (i.e., uptake of a COVID-19 vaccine). Our findings suggest, for example, that a "one size fits all" approach to mass media interventions represents, at best, a partial solution to increasing vaccination uptake and, at worst, a solution that backfires, amplifying existing inequalities. These findings suggest that future interventions need to be targeted to a range of sub-populations and diverse communities [23,24]. The range of interventions should offer different kinds of educational, persuasive, or enabling approaches [25] to different groups of people and there is growing recognition that such interventions should be co-produced. Issues of visual cultural representation, identifiable local key opinion 
leaders, readability and reading levels, and trust will all be important considerations. These emerging findings also lend themselves to considering the wider opportunities for targeting afforded by social media. Whilst marketing and politics are capitalising on these new opportunities, it is less clear if and how public health can do so. Equally, these initial findings also suggest that health care professionals should be trained to anticipate and respond to diverse segments of the population differently, with overt and inclusive demonstrations of cultural competencies.

If we imagine a future of targeted interventions to improve COVID-19 vaccination uptake, it is also important to focus now on the granularity of messaging that will shortly be needed. Whilst evidence is emerging concerning where and amongst whom interventions need to be targeted, it is currently unclear how intervention content should be tailored to these populations and communities and their particular beliefs. We need to use the full range of social and behavioural sciences now to address this key gap and ensure so that we are prepared and ready to support the whole population in gaining the maximum benefit from available vaccines. One preliminary study in this area used the behaviour change wheel [26-28] to provide recommendations for the design of interventions aimed at maximising public acceptance of the COVID-19 vaccine. The findings suggested that interventions should utilise the behaviour change techniques [29] of information about health, emotional, social, and environmental consequences, and salience of consequences in order to provide the public with information about the beneficial consequences of vaccination for themselves and for others [30]. Further, more in-depth research that focuses on intervention development, such as this study, is required.

Strengths of the current study include the large sample size, the use of two waves of data collection to examine stability in COVID-19 vaccination intention, and the inclusion of a range of sociodemographic factors to allow us to understand the social patterning of COVID-19 vaccination acceptance. However, it is important to note that the study was not nationally representative, and weighting was applied to the gender and education variables due to the underrepresentation of males and those with lower levels of education. Furthermore, we experienced a participant drop-out rate of $41 \%$ from Time 1 to Time 2 meaning that we could not draw any conclusions about the change in COVID-19 vaccination intention from those participants who were lost at follow-up. Moreover, we experienced higher dropout in those with lower education levels, males, and in the younger age group. In addition, participants were answering about their intention to accept a hypothetical COVID-19 vaccine, without information regarding the specifics of the vaccine in terms of number of doses needed, potential side-effects, or prioritisation of delivery across the population. All of which may influence eventual uptake of a COVID-19 vaccination.

\section{Conclusions}

Intention to accept a COVID-19 vaccine is currently high in Scotland and our findings suggest that intention to receive the vaccine did not fall in the context of lower infection rates and fewer restrictions. However, the data also point to a sizeable minority of the public who are hesitant about receiving a future COVID-19 vaccine. Of note, intention was higher in participants of white ethnicity as compared with those from BAME groups, and in those with higher levels of income and education. Our findings and those from other studies suggest that future interventions need to be targeted at a range of subpopulations and diverse communities. To do so, we need to better understand the barriers to vaccination in these groups so that we can collectively be better prepared to deliver appropriate evidence-based culturally and community-appropriate messaging aimed at maximising COVID-19 vaccine uptake.

Author Contributions: Conceptualization, L.W., P.F., C.P.T.; methodology, L.W. and L.R.; formal analysis, J.M. and D.Y.; investigation, L.R.; writing—original draft preparation, L.W., P.F., and J.M.; writing-review and editing, all authors.; funding acquisition, L.W., P.F., C.P.T. The C.P.T.: Leanne Fleming, Xanne Janssen, Alison Kirk, Madeleine Grealy, Bradley MacDonald. All authors have read and agreed to the published version of the manuscript. 
Funding: This research was funded by the Chief Scientist Office in Scotland (ref COV/SCL/20/09).

Institutional Review Board Statement: The study was conducted according to the guidelines of the Declaration of Helsinki, and approved by the Ethics Committee) of The University of Strathclyde (ref. 61/05/05/2020/A Williams on 12 May 2020).

Informed Consent Statement: Informed consent was obtained from all participants involved in the study.

Data Availability Statement: The data presented in this study are openly available in The Open Science Framework, https:/ / osf.io/tn7v9/ doi:10.17605/OSF.IO/TN7V9.

Conflicts of Interest: The authors declare no conflict of interest. The funders had no role in the design of the study; in the collection, analyses, or interpretation of data; in the writing of the manuscript, or in the decision to publish the results.

\section{References}

1. De Figueiredo, A.; Simas, C.; Karafillakis, E.; Paterson, P.; Larson, H.J. Mapping global trends in vaccine confidence and investigating barriers to vaccine uptake: A large-scale retrospective temporal modelling study. Lancet 2020, 396, 898-908. [CrossRef]

2. Larson, H.J.; De Figueiredo, A.; Xiahong, Z.; Schulz, W.S.; Verger, P.; Johnston, I.G.; Jones, N.S. The state of vaccine confidence 2016: Global insights through a 67-country survey. EBioMedicine 2016, 12, 295-301. [CrossRef]

3. Wellcome Trust. Wellcome Global Monitor. 2018. Available online: https://wellcome.ac.uk/reports/wellcome-global-monitor/ 2018 (accessed on 20 November 2020).

4. Jorgensen, P.; Mereckiene, J.; Cotter, S.; Johansen, K.; Tsolova, S.; Brown, C. How close are countries of the WHO European Region to achieving the goal of vaccinating $75 \%$ of key risk groups against influenza? Results from national surveys on seasonal influenza vaccination programmes, 2008/2009 to 2014/2015. Vaccine 2018, 36, 442-452. [CrossRef]

5. MacDonald, N.E. The SAGE Working Group on Vaccine Hesitancy. Vaccine hesitancy: Definition, scope and determinants. Vaccine 2015, 33, 4161-4164. [CrossRef]

6. Lane, S.; MacDonald, N.E.; Marti, M.; Dumolard, L. Vaccine hesitancy around the globe: Analysis of three years of WHO/UNICEF Joint Reporting Form data-2015-2017. Vaccine 2008, 36, 3861-3867. [CrossRef] [PubMed]

7. Larson, H.J.; Jarrett, C.; Eckersberger, E.; Smith, D.M.D.; Paterson, P. Understanding vaccine hesitancy around vaccines and vaccination from a global perspective: A systematic review of published literature, 2007-2012. Vaccine 2014, 32, 2150-2159. [CrossRef] [PubMed]

8. Brewer, N.T.; Chapman, G.B.; Rothman, A.J.; Leask, J.; Kempe, A. Increasing vaccination: Putting psychological science into action. Psychol. Sci. Public Interest 2017, 18, 149-207. [CrossRef] [PubMed]

9. Schmid, P.; Rauber, D.; Betsch, C.; Lidolt, G.; Denker, M.L. Barriers of influenza vaccination intention and behavior-a systematic review of influenza vaccine hesitancy, 2005-2016. PLoS ONE 2017, 12, e0170550. [CrossRef] [PubMed]

10. Bish, A.; Yardley, L.; Nicoll, A.; Michie, S. Factors associated with uptake of vaccination against pandemic influenza: A systematic review. Vaccine 2011, 29, 6472-6484. [CrossRef] [PubMed]

11. Brien, S.; Kwong, J.C. The determinants of 2009 pandemic A/H1N1 influenza vaccination: A systematic review. Vaccine 2012, 30, 1255-1264. [CrossRef]

12. Fabry, P.; Gagneur, A.; Pasquier, J.-C. Determinants of A(H1N1) vaccination: Cross sectional study in a population of pregnant women in Quebec. Vaccine 2011, 29, 1824-1829. [CrossRef] [PubMed]

13. Han, K.Y.J.; Michie, S.; Potts, H.W.; Rubin, G.J. Predictors of influenza vaccine uptake during the 2009/10 influenza A H1N1v ('swine flu') pandemic: Results from five national surveys in the United Kingdom. Prev. Med. 2016, 84, 57-61. [CrossRef] [PubMed]

14. Seale, H.; Heywood, A.E.; McLaws, M.L.; Ward, K.F.; Lowbridge, C.P.; Van, D.; MacIntyre, C.R. Why do I need it? I am not at risk! Public perceptions towards the pandemic (H1N1) 2009 vaccine. BMC Infect. Dis. 2010, 99. [CrossRef]

15. Lazarus, J.V.; Ratzan, S.C.; Palayew, A.; Gostin, L.O.; Larson, H.J.; Rabin, K.; Kimball, S.; El-Mohandes, A. A global survey of potential acceptance of a COVID-19 vaccine. Nat. Med. 2020. [CrossRef] [PubMed]

16. Rhodes, A.; Hoq, M.; Measey, M.-A.; Danchin, M. Intention to vaccinate against COVID-19 in Australia. Lancet Infect. Dis. 2020. [CrossRef]

17. Malik, A.M.; McFadden, S.M.; Elharake, J.; Omer, S.B. Determinants of COVID-19 vaccine acceptance in the US. EClinical Med. 2020. [CrossRef]

18. IPSOS MORI: News Three in Four Adults Globally Say They Would Get a Vaccine for COVID-19. Available online: https:// www.ipsos.com/ipsos-mori/en-uk/three-four-adults-globally-say-they-would-get-vaccine-covid-19 (accessed on 10 November 2020).

19. The COCONEL Group. A future vaccination campaign against COVID-19 at risk of vaccine hesitancy and politicisation. Lancet Infect. Dis. 2020, 20, 769-770. [CrossRef]

20. Dodd, R.H.; Cvejic, E.; Bonner, C.; Pickles, K.; McCaffery, K.J. Sydney Health Literacy Lab COVID-19 group. Willingness to vaccinate against COVID-19 in Australia. Lancet Infect. Dis. 2020. [CrossRef] 
21. Bartsch, S.M.; O'Shea, K.J.; Ferguson, M.C.; Bottazzi, M.E.; Wedlock, P.T.; Strych, U.; McKinnell, J.A.; Siegmund, S.S.; Cox, S.N.; Hotez, P.J.; et al. Vaccine efficacy needed for a COVID-19 coronavirus vaccine to prevent or stop an epidemic as the sole intervention. Am. J. Prev. Med. 2020, 59, 493-503. [CrossRef]

22. Office for National Statistics Deaths involving COVID-19 by Local Area and Socioeconomic Deprivation: Deaths Occurring between 1 March and 31 July 2020. Available online: https://www.ons.gov.uk/peoplepopulationandcommunity/ birthsdeathsandmarriages / deaths/bulletins/deathsinvolvingcovid19bylocalareasanddeprivation/deathsoccurringbetween1 marchand31july2020 (accessed on 12 November 2020).

23. Dube, E.; Leask, J.; Wolff, B.; Hickler, B.; Balaban, V.; Hosein, E.; Habersaat, K. The WHO Tailoring Immunization Programmes (TIP) approach: Review of implementation to date. Vaccine 2018, 36, 1509-1515. [CrossRef]

24. Thomson, A.; Vallée-Tourangeau, G.; Suggs, L.S. Strategies to increase vaccine acceptance and uptake: From behavioral insights to context-specific, culturally-appropriate, evidence-based communications and interventions. Vaccine 2018, 36, 6457-6458. [CrossRef] [PubMed]

25. Dube, E.; Gagnon, D.; MacDonald, N.E. The SAGE Working Group on Vaccine Hesitancy. Strategies intended to address vaccine hesitancy: Review of published reviews. Vaccine 2015, 33, 4191-4203. [CrossRef] [PubMed]

26. Michie, S.; Atkins, L.; West, R. The Behaviour Change Wheel: A Guide to Designing Interventions; Silverback: London, UK, 2014.

27. Michie, S.; Stralen, M.M.; West, R. The behaviour change wheel: A new method for characterising and designing behaviour change interventions. Implement. Sci. 2011, 6, 42. [CrossRef] [PubMed]

28. Cane, J.; O'Connor, D.; Michie, S. Validation of the theoretical domains framework for use in behaviour change and implementation research. Implement. Sci. 2012, 7, 37. [CrossRef] [PubMed]

29. Michie, S.; Richardson, M.; Johnston, M.; Abraham, C.; Francis, J.; Hardeman, W.; Wood, C.E. The behavior change technique taxonomy (v1) of 93 hierarchically clustered techniques: Building an international consensus for the reporting of behavior change interventions. Ann. Behav. Med. 2013, 46, 81-95. [CrossRef] [PubMed]

30. Williams, L.; Gallant, A.J.; Rasmussen, S.; Brown Nicholls, L.A.; Cogan, N.; Deakin, K.; Young, D.; Flowers, P. Towards intervention development to increase the uptake of COVID-19 vaccination among those at high risk: Outlining evidence-based and theoretically informed future intervention content. Br. J. Health Psychol. 2020, 25, 1039-1054. [CrossRef] [PubMed] 